\title{
LAYER POTENTIALS FOR ELASTOSTATICS AND HYDROSTATICS IN CURVILINEAR POLYGONAL DOMAINS
}

\author{
JEFF E. LEWIS
}

\begin{abstract}
The symbolic calculus of pseudodifferential operators of Mellin type is applied to study layer potentials on a plane domain $\Omega^{+}$whose boundary $\partial \Omega^{+}$is a curvilinear polygon. A "singularity type" is a zero of the determinant of the matrix of symbols of the Mellin operators and can be used to calculate the "bad values" of $p$ for which the system is not Fredholm on $L^{p}\left(\partial \Omega^{+}\right)$.

Using the method of layer potentials we study the singularity types of the system of elastostatics

$$
L \mathbf{u}=\mu \Delta \mathbf{u}+(\lambda+\mu) \nabla \operatorname{div} \mathbf{u}=0 .
$$

in a plane domain $\Omega^{+}$whose boundary $\partial \Omega^{+}$is a curvilinear polygon. Here $\mu>0$ and $-\mu \leq \lambda \leq+\infty$. When $\lambda=+\infty$, the system is the Stokes system of hydrostatics. For the traction double layer potential, we show that all singularity types in the strip $0<\operatorname{Re} z<1$ lie in the interval $\left(\frac{1}{2}, 1\right)$ so that the system of integral equations is a Fredholm operator of index 0 on $L^{p}\left(\partial \Omega^{+}\right)$for all $p$, $2 \leq p<\infty$. The explicit dependence of the singularity types on $\lambda$ and the interior angles $\theta$ of $\partial \Omega^{+}$is calculated; the singularity type of each corner is independent of $\lambda$ iff the corner is nonconvex.
\end{abstract}

\section{INTRODUCTION}

Recently there has been considerable interest in using layer potentials to solve $L^{p}$ boundary value problems for elliptic operators and systems on a Lipschitz domain $\Omega^{+}$in $\mathbf{R}^{n}$. For the systems of elastostatics [DKV] and hydrostatics [FKV], Dahlberg, Fabes, Kenig, and Verchota have used Rellich type identities to prove that the double layer potential integral equations yield a Fredholm operator of index 0 on $L^{2}\left(\partial \Omega^{+}\right)$. For $p \neq 2$ only limited information is available on the boundary integral equations for general Lipschitz domains in $\mathbf{R}^{n}$. The general problem of the notion of symbol on the boundary of a general Lipschitz domain is still very much open.

In this paper we treat a very special case: a curvilinear polygonal domain in $\mathbf{R}^{2}$. In this 2-dimensional case a precise symbolic calculus of pseudodifferential operators of Mellin type is available. We show that certain double layer boundary integral equations yield operators which for all $p, 2 \leq p<\infty$, are

Received by the editors December 14, 1988.

1980 Mathematics Subject Classification (1985 Revision). Primary 35J25, 45E05.

Research partially supported by the Italian CNR. 
Fredholm operators of index 0 on $L^{p}\left(\partial \Omega^{+}\right)$. The singularities exhibited for $p<2$ show the limitations of the general theory.

We develop the theory of double layer potentials for treating boundary value problems for second order elliptic systems in a plane domain $\Omega^{+}$which is bounded by a curvilinear polygon $\partial \Omega^{+}$. The double layer potential operators on $L^{p}\left(\partial \Omega^{+}\right)$are interpreted as systems of pseudodifferential operators of Mellin type, or more simply Mellin operators, on $L^{p}(0,1)$. A symbolic calculus for Mellin operators was developed by Lewis and Parenti [LP] and J. Elschner [E]. Our particular interest is to explicitly calculate the singularity types. A singularity type of a system of Mellin operators $\mathbf{K}$ is defined as a complex number $z_{0}, \operatorname{Re} z_{0}=\frac{1}{p}$, at which the determinant of the principal symbol, $\operatorname{Smbl}^{\frac{1}{p}}(\mathbf{K})$, vanishes. Elschner $[E]$ has used singularity types to construct parametrices and develop asymptotic expansions for solutions of the equation $\mathbf{K} \mathbf{f}=\mathbf{g}$. For a different approach to a symbol map on curves with corners, see Costabel [C].

In $\S 1$ we describe the algebra of Mellin operators on the finite interval $J \equiv$ $[0,1]$. We follow closely the notation of $[E]$ since the parametrices have meromorphic symbols with poles at the singularity types.

In $\S 2$ we describe a class of double layer kernel operators and show that they are examples of Mellin operators; their principal symbols are calculated.

$\S 3$ gives a parametrization of a curvilinear polygon $\partial \Omega^{+}$which reduces a system of double layer potential integral operators on $L^{p}\left(\partial \Omega^{+}\right)$to a big system of operators of Mellin type on $L^{p}(J)$. The part of the symbol arising from each vertex $P_{k}$ of $\partial \Omega^{+}$is the same as for the corresponding operator in a plane sector of interior opening $\theta_{k}$. Theorem 2 shows that the "bad values" of $p$ for which the operators are not Fredholm on $L^{p}\left(\partial \Omega^{+}\right)$are the same as for the sector problems; for the "good values" of $p$, the index of the system on $L^{p}\left(\partial \Omega^{+}\right)$can be calculated from the change in argument of the principal symbol for the sector problems and Theorem 1 yields the index. Theorem 2 should be considered as a localization result.

In $\S 4$ we apply our results to for the system of linear elastostatics:

$$
L \mathbf{u}=\mu \Delta \mathbf{u}+(\lambda+\mu) \nabla \operatorname{div} \mathbf{u}=0 .
$$

The numbers $\mu$ and $\lambda$ are the Lamé moduli; we assume $\mu>0$ and that $-\mu \leq \lambda \leq+\infty$. When $\lambda=-\mu$, the operator $L$ is two copies of the Laplace operator; when $\lambda=+\infty$, we interpret the operator as the Stokes system of hydrostatics:

$$
\left\{\begin{array}{l}
L(\mathbf{u}, p)=\mu \Delta \mathbf{u}-\nabla p=0, \\
\operatorname{div} \mathbf{u}=0 .
\end{array}\right.
$$

Our interest is in the description of the singularities of solutions in terms of the interior angles $\theta$ at the vertices of $\partial \Omega^{+}$and the parameter $\lambda$. We state our results in terms of the normalized parameter $b$, defined as

$$
b=\frac{\lambda+\mu}{\lambda+2 \mu},
$$

so that $0 \leq b \leq 1$. 
The boundary operator of physical significance is the traction operator. The stress tensor $\mathbf{T}=\left(T_{i, k}\right)$ is defined by

$$
T_{i, k}(\mathbf{u})=\lambda(\operatorname{div} \mathbf{u}) \delta_{i, k}+\mu\left(u_{i, k}+u_{k, i}\right),
$$

or in the case of the Stokes system $(\lambda=+\infty)$,

$$
T_{i, k}(\mathbf{u}, p)=-p(x) \delta_{i, k}+\mu\left(u_{i, k}+u_{k, i}\right),
$$

where $u_{i, k}=\partial u_{i} / \partial x_{k}$. If $\vec{\nu}$ is the outward normal to $\Omega^{+}$at a point $P \in \partial \Omega^{+}$, the traction operator is

$$
\mathbf{T}_{\vec{\nu}}(\mathbf{u})=\mathbf{T}(\mathbf{u}) \vec{\nu}
$$

We shall also consider another conormal boundary operator

$$
\mathbf{N}_{\vec{\nu}}(\mathbf{u})=\mu \frac{\partial \mathbf{u}}{\partial \vec{\nu}}+(\lambda+\mu)(\operatorname{div} \mathbf{u}) \vec{\nu},
$$

which for $b=0$ reduces to the Neumann boundary operator. Let $\Omega^{-}$denote the complement of $\Omega^{+} \cup \partial \Omega^{+}$. The boundary value problems we shall treat are

(1) The Dirichlet problems $D_{ \pm}$:

$$
\left\{\begin{array}{l}
L \mathbf{u}=0 \quad \text { in } \Omega^{ \pm} \\
\left.\mathbf{u}\right|_{\partial \Omega^{ \pm}}=\mathbf{g} \in L^{p}\left(\partial \Omega^{+}\right)
\end{array}\right.
$$

(2) The traction problems $T_{ \pm}$:

$$
\left\{\begin{array}{l}
L \mathbf{u}=0 \quad \text { in } \Omega^{ \pm}, \\
\left.\mathbf{T}_{\vec{\nu}}(\mathbf{u})\right|_{\partial \Omega^{ \pm}}=\mathbf{g} \in L^{p}\left(\partial \Omega^{+}\right) .
\end{array}\right.
$$

(3) The Neumann problems $N_{ \pm}$:

$$
\left\{\begin{array}{l}
L \mathbf{u}=0 \text { in } \Omega^{ \pm} \\
\left.\mathbf{N}_{\vec{\nu}}(\mathbf{u})\right|_{\partial \Omega^{ \pm}}=\mathbf{g} \in L^{p}\left(\partial \Omega^{+}\right) .
\end{array}\right.
$$

We represent the solutions of $D_{ \pm}$as double layer potentials and the solutions of $T_{ \pm}$and $N_{ \pm}$as single layer potentials using the fundamental solution given by Kupradze [K, Chapter 9, (9.2)]:

$$
\Gamma(X)=\left(\Gamma_{i, j}(X)\right)=\left(\delta_{i, j} \frac{n}{2 \pi} \log r^{2}-\frac{m}{\pi} \frac{x_{i} x_{j}}{r^{2}}\right),
$$

with $r^{2}=x_{1}^{2}+x_{2}^{2}$ and

$$
n=\frac{\lambda+3 \mu}{2 \mu(\lambda+2 \mu)}, \quad m=\frac{\lambda+\mu}{2 \mu(\lambda+2 \mu)} .
$$

This fundamental solution satisfies

$$
L(\boldsymbol{\Gamma}(X))=2 \delta(X) \mathbf{I},
$$


where the operator $L$ is applied to the columns of the matrix $\Gamma$. When $b=$ 1, we have $n=m$ and as in Ladyzhenskaya [La, Chapter 3] introduce the fundamental pressure (row) vector:

$$
\mathbf{q}(X)=\frac{1}{\pi} \frac{X}{r^{2}}
$$

so that $\{\boldsymbol{\Gamma}, \mathbf{q}\}$ is a solution of the adjoint Stokes system

$$
\left\{\begin{array}{l}
\mu \Delta \boldsymbol{\Gamma}+\nabla \mathbf{q}=2 \delta(X) \mathbf{I}, \\
\operatorname{div} \boldsymbol{\Gamma}=0 .
\end{array}\right.
$$

The solution of $D_{ \pm}$is sought in the form of the double layer potential

$$
\mathbf{u}_{\mathbf{T}}(X)=\int_{\partial \Omega^{+}} \mathbf{T}_{\vec{\nu}(Q)}(\boldsymbol{\Gamma}(X-Q)) \mathbf{f}(Q) d \sigma_{Q^{\prime}}
$$

Taking nontangential limits in $L^{p}\left(\partial \Omega^{+}\right)$from inside and outside $\Omega^{+}$, and calling the resulting limits $\mathbf{u}_{\mathrm{T}}^{ \pm}$, we obtain

$$
(0-18) \quad \mathbf{u}_{\mathbf{T}}^{ \pm}(P) \equiv \mathbf{K}_{\mathbf{T}}^{ \pm} \mathbf{f}(P)= \pm \mathbf{I f}(P)+\text { p.v. } \int_{\partial \Omega^{+}} \mathbf{T}_{\vec{\nu}(Q)}(\boldsymbol{\Gamma}(P-Q)) \mathbf{f}(Q) d \sigma_{Q},
$$

where even in the case where $\partial \Omega^{+}$is flat the integral operator in $(0-18)$ is not compact.

In a like manner the solutions of $T_{ \pm}$and $N_{ \pm}$are represented in the form of a single layer potential

$$
\mathbf{u}_{\mathbf{S}}(X)=-\int_{\partial \Omega^{+}} \Gamma(X-Q) \mathbf{f}(Q) d \sigma_{Q}
$$

Applying the boundary operators $\mathbf{T}_{ \pm}$and $\mathbf{N}_{ \pm}$to $\mathbf{u}_{\mathbf{S}}$ we obtain integral equations which are adjoints to the double layer integral equations; e.g.,

$$
\left[\mathbf{T}_{ \pm}\left(\mathbf{u}_{\mathbf{S}}\right) \vec{\nu}\right](P)=\left(\mathbf{K}_{\mathbf{T}}^{\mp}\right)^{*} \mathbf{f}(P) .
$$

In $\S 4$ we give explicit expressions for the kernels for elastostatics and hydrostatics in a plane sector.

In $\S 5$ we compute the symbols for the problems in a plane sector. Theorem 7 gives a very simple expression for the determinant of the matrix of symbols in terms of the parameter $b$ and the interior angle $\theta$.

In $\S 6$, we calculate the singularity types of $\mathbf{K}_{\mathbf{T}}^{ \pm}$. We first summarize the results in a a plane sector in Theorem 8 . Theorem 8 shows that there is a contrast in the cases of a corner of $\Omega^{+}$where $\Omega^{+}$is convex $(0<\theta<\pi)$, and the case of a reentrant corner $(\pi<\theta<2 \pi)$. We first note that when $b=0$, the operator $\mathbf{T}_{\vec{\nu}}$ does not cover $L$; however, $\mathbf{N}_{\vec{\nu}}$ covers $L$ for $0 \leq b \leq 1$. The nature of the singularity types is

\footnotetext{
${ }^{1}$ In the case $b=1$, the kernel $\mathbf{T}_{\vec{\nu}(Q)}(\boldsymbol{\Gamma}(X-Q))$ is replaced by

$$
\mathbf{T}_{\vec{\nu}(Q)}^{\prime}(\boldsymbol{\Gamma}(X-Q), \mathbf{q}) \equiv\left(\mathbf{q} \delta_{i, k}+\mu\left(\Gamma_{i, k}+\Gamma_{k, i}\right)\right) \vec{\nu}(Q),
$$
}

the stress tensor being applied to the columns of $\{\boldsymbol{\Gamma}, \mathbf{q}\}$. 
Case I. For $0<\theta<\pi$, the Mellin operators $\mathbf{K}_{\mathbf{T}}^{+}$and $\mathbf{K}_{\mathbf{N}}^{+}$have the same singularities for $0<b \leq 1$. For $0<b<1$, there are two singularity types in the strip $0<\operatorname{Re} z<1$; both singularity types are real and lie in $\left(\frac{1}{2}, 1\right)$. When $b=1$, there is a value $\gamma_{\text {crit }} \approx 257^{\circ} 27^{\prime}$ for which there are two singularity types for $0<\theta<2 \pi-\gamma_{\text {crit }}$; for $2 \pi-\gamma_{\text {crit }} \leq \theta<\pi$, there is only one singularity type in the strip.

Case II. For $\pi<\theta<2 \pi$, the singularity types for $\mathbf{K}_{\mathbf{T}}^{+}$in the strip $0<\operatorname{Re} z<1$ are independent of $b$, lie in $\left(\frac{1}{2}, 1\right)$ and approach $\frac{1}{2}$ as $\theta$ approaches $2 \pi$; there is one singularity type in the strip for $\pi<\theta \leq \gamma_{\text {crit }}$; a second singularity type develops for $\gamma_{\text {crit }}<\theta<2 \pi$.

Finally, Theorem 9 summarizes the "good values" and "bad values" of $p$ for the double layer potential integral equations on $L^{p}\left(\partial \Omega^{+}\right)$, where $\partial \Omega^{+}$is a curvilinear polygon.

\section{MEllin operators on a Finite interval}

Algebras of Mellin operators on $J \equiv[0,1]$ are defined in [LP, Definition (4.1)] and [E, Definition (4.1)]. We follow closely the notions of [E] since Elschner develops an extension to meromorphic symbols which arise in constructing parametrices. For $0 \leq \alpha<\beta \leq 1$, define the strip $\Gamma_{\alpha, \beta}=\{z \in \mathbf{C}$ : $\alpha<\operatorname{Re} z<\beta\}$, and let $\Gamma_{\gamma}$ be the line $\{z=\gamma+i \xi:-\infty \leq \xi \leq+\infty\}$. The symbol space $\tilde{\Sigma}_{\alpha, \beta}^{0}$ is defined in [E, Definition (1.12)].

For $f \in C_{0}^{\infty}\left(\mathbf{R}^{+}\right)$define the Mellin transform of $f$ by

$$
\mathscr{M} f(z)=\tilde{f}(z)=\int_{0}^{\infty} t^{z-1} f(t) d t
$$

Let $\partial=-t d / d t$, and for $a \in \tilde{\Sigma}_{\alpha, \beta}^{0}$, we define the Mellin operator $a(t, \partial) \in$ Op $\tilde{\Sigma}_{\alpha, \beta}^{0}$ by

$$
a(t, \partial) f(t)=\frac{1}{2 \pi i} \int_{\operatorname{Re} z=\gamma} t^{-z} a(t, z) \tilde{f}(z) d z,
$$

with $\gamma \in(\alpha, \beta)$.

If $f \in L^{p}(J)$ let $R f$ be the reflection

$$
R f(t)=f(1-t) .
$$

Definition 1.1. An operator A from $C_{0}^{\infty}(J)$ to $C^{\infty}(J)$ is a Mellin operator in the class $\mathrm{Op} \Sigma_{\alpha, \beta}(J)$ iff

(1) For all $\phi, \psi \in C_{0}^{\infty}([0,1))$, there are operators $a_{0 \phi \psi}(t, \partial) \in \mathrm{Op} \tilde{\Sigma}_{\alpha, \beta}^{0}$ and $C_{0 \phi \psi}$, compact on $L^{p}(J)$ for all $p$ with $\frac{1}{p} \in(\alpha, \beta)$, such that

$$
\phi A \psi=a_{0 \phi \psi}(t, \partial)+C_{0 \phi \psi} \text {. }
$$

(2) If $\phi, \psi \in C^{\infty}([0,1])$ have disjoint supports, the operator $\phi A \psi$ is compact on $L^{p}(J), \frac{1}{p} \in(\alpha, \beta)$.

(3) The operator $A^{R} \equiv R A R$ satisfies conditions (1) and (2). 
To define the principal symbol, $\operatorname{Smbl}^{\frac{1}{p}}(A)$, for $A$ as an operator on $L^{p}(J)$, we use that there are uniquely defined functions $a_{0}(z), a_{0 \pm}(t)$ such that for all $\phi, \psi \in C_{0}^{\infty}([0,1))$,

$$
\begin{aligned}
a_{0 \phi \psi}(0, z) & =\phi(0) a_{0}(z) \psi(0), & & z \in \Gamma_{\alpha, \beta}, \\
a_{0 \phi \psi}\left(t, \frac{1}{p} \pm i \infty\right) & =\phi(t) a_{0 \pm}(t) \psi(t), & & 0 \leq t<1, \frac{1}{p} \in(\alpha, \beta) .
\end{aligned}
$$

There are uniquely defined functions $a_{1}(z), a_{1 \pm}(t)$ such that for all $\phi, \psi \in$ $C_{0}^{\infty}([0,1))$,

$$
\begin{aligned}
\left(a^{R}\right)_{0 \phi \psi}(0, z) & =\phi(0) a_{1}(z) \psi(0), & & z \in \Gamma_{\alpha, \beta}, \\
\left(a^{R}\right)_{0 \phi \psi}\left(t, \frac{1}{p} \pm i \infty\right) & =\phi(t) a_{1 \pm}(t) \psi(t), & & 0 \leq t<1, \frac{1}{p} \in(\alpha, \beta) .
\end{aligned}
$$

Moreover

$$
a_{0 \pm}(t)=a_{1 \mp}(1-t), \quad 0<t<1 .
$$

Let $\mathscr{R}_{J}^{\frac{1}{p}}$ be the oriented boundary of the rectangle:

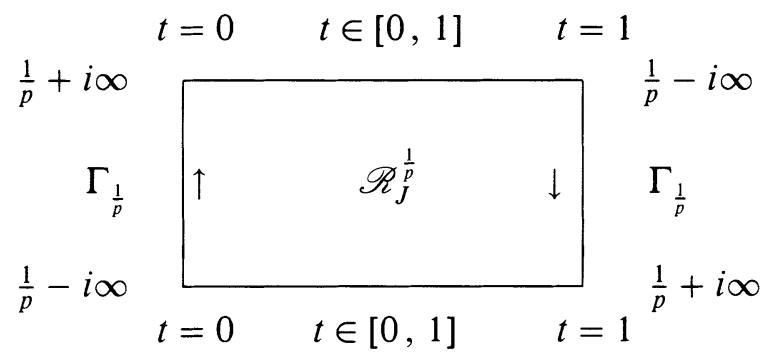

Definition 1.2. Let $A \in \mathrm{Op} \Sigma_{\alpha, \beta}(J)$ and $\frac{1}{p} \in(\alpha, \beta)$. The principal symbol of $A$ as an operator on $L^{p}(J), \operatorname{Smbl}^{\frac{1}{p}}(A)$, is the quadruple of functions $a_{0}\left(\frac{1}{p}+i \xi\right)$, $a_{0+}(t)=a_{1-}(1-t), a_{1}\left(\frac{1}{p}+i \xi\right), a_{0-}(t)=a_{1+}(1-t)$, considered as a continuous function on $\mathscr{R}_{J}^{\frac{1}{p}}$ :

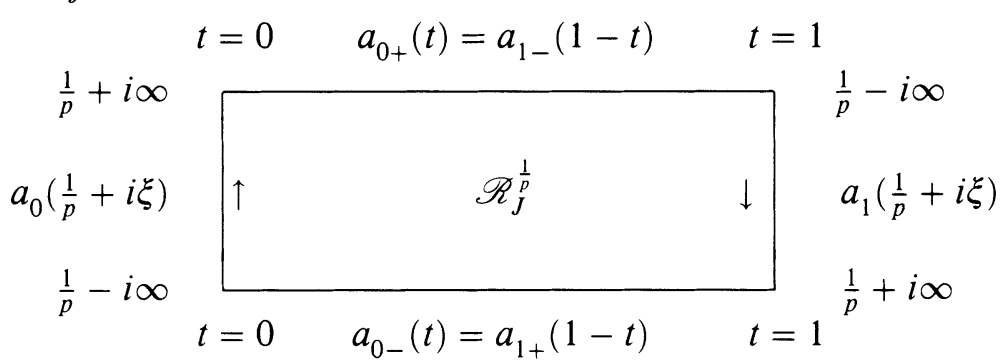

Definition 1.3. Let $A=\left(A_{i j}\right)$ be an $N \times N$ matrix of operators in $\operatorname{Op} \Sigma_{\alpha, \beta}(J)$. The system $A$ is elliptic on $L^{p}(J)^{2}$ iff $\mathrm{Smbl}^{\frac{1}{p}} A$ is a nonsingular matrix on $\mathscr{R}_{J}^{\frac{1}{p}}$. A number $z_{0} \in \Gamma_{\alpha, \beta}$ is a singularity type for $A$ at $t=0[t=1]$ if

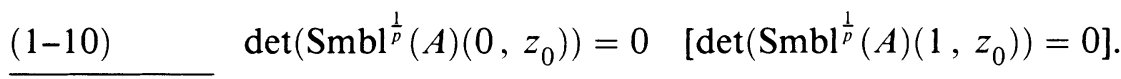

\footnotetext{
${ }^{2}$ For brevity we write $L^{p}(J)$ for $\left[L^{p}(J)\right]^{N}$.
} 
The following is shown in [E, Theorems 4.4 and 4.6] and [LP, Theorems 4.1 and 4.2].

Theorem 1. Let $A=\left(A_{i j}\right)$ be an $N \times N$ matrix of operators in $\mathrm{Op} \Sigma_{\alpha, \beta}(J)$. Then

(1) $A$ is a Fredholm operator on $L^{p}(J)$ iff $A$ is elliptic on $L^{p}(J)$.

(2) If $A$ is elliptic on $L^{p}(J)$, define

$(1-11) \quad \operatorname{ind}_{p}(A)=\operatorname{dim}\left((\operatorname{ker} A) \cap L^{p}(J)\right)-\operatorname{dim}\left(\left(\operatorname{ker} A^{*}\right) \cap L^{p / p-1}(J)\right)$.

Then

$$
\operatorname{ind}_{p}(A)=\frac{1}{2 \pi} \Delta_{\mathscr{R}_{J}^{\frac{1}{p}}}\left\{\arg \left(\operatorname{det}\left(\operatorname{Smbl}^{\frac{1}{p}} A\right)\right)\right\}
$$

where the change in arg is taken as $\mathscr{R}_{J}^{\frac{1}{p}}$ is traversed in the clockwise direction.

Remark. In treating boundary value problems in domains with corners it is useful to regard Mellin operators as acting on weighted spaces, e.g., $L^{p, \sigma}(J) \equiv$ $\left\{f: t^{\sigma} f(t) \in L^{p}(J)\right\}$. In this case we suppose that both $\frac{1}{p}+\sigma$ and $\frac{1}{p}$ lie in $(\alpha, \beta)$. The principal symbol would be defined on the oriented rectangle $\mathscr{R}_{J}^{\frac{1}{p}+\sigma, \frac{1}{p}}$ whose left-hand side is the contour $\Gamma_{\frac{1}{p}+\sigma}$, and whose right-hand side is the contour $\Gamma_{\frac{1}{p}}$. Cf. [E], but note that our notation differs slightly from [E, (4.8) ff.]. The approach of weighted spaces is especially useful where different weights may be introduced at different vertices of a polygon.

When double layer potentials on a curvilinear polygon $\partial \Omega^{+}$are reduced to a system of Mellin operators as in $\S 3$, the operators near $t=1$ will correspond to a smooth part of $\partial \Omega^{+}$so that singularities at $t=1$ will not appear; the change in arg of $\operatorname{det}\left(\operatorname{Smbl}^{\frac{1}{p}} A\right)$ will occur entirely on the contour $\Gamma_{\frac{1}{p}}$ on the left-hand side of (1-8).

\section{EXAMPLES OF Mellin OPERATORS}

In this section we give examples of Mellin operators in $O p \Sigma_{0,1}(J)$.

1. The finite Hilbert transform $H$ is defined by

$$
H f(t)=\text { p.v. } \frac{1}{\pi} \int_{0}^{1} \frac{f(s)}{t-s} d s .
$$


$H$ is in Op $\Sigma_{0,1}(J)$ and $\operatorname{Smbl}^{\frac{1}{p}} H$ is

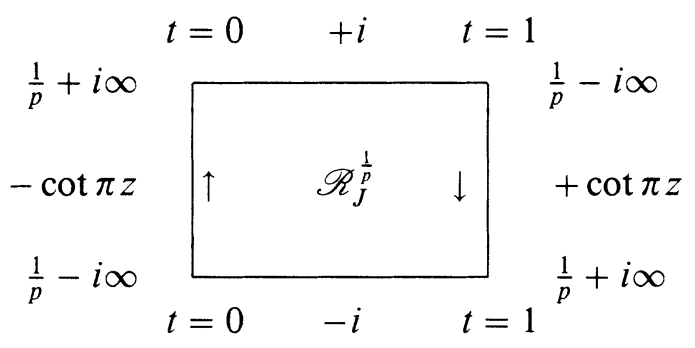

2. Let $k(t) \in \mathscr{F}_{-\infty, 1}^{\prime}$ [LP, Definition 1.1]; i.e., $k(t) \in C^{\infty}([0, \infty))$ and for every $l \geq 0, \delta>0, \partial^{l} k(t)=O\left(t^{-1+\delta}\right)$ as $t \rightarrow \infty$. Define the Hardy kernel operator by

$$
K f(t)=\int_{0}^{1} k\left(\frac{t}{s}\right) f(s) \frac{d s}{s} .
$$

Then $K \in \mathrm{Op} \Sigma_{0,1}(J)$ and $\mathrm{Smbl}^{\frac{1}{p}} K$ is

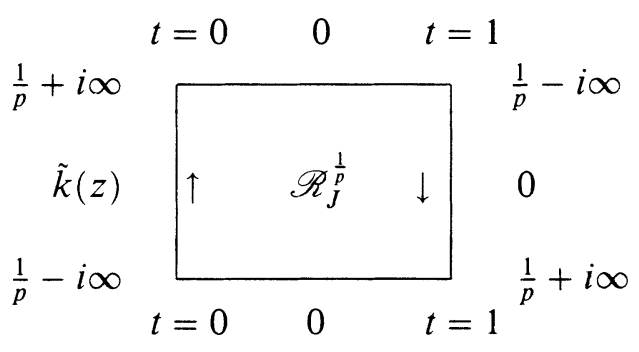

Definition 2.1. A function $k(x, y)$ is a double layer kernel if

(1) $k \in C^{\infty}\left(\mathbf{R}^{2} \backslash\{0\}\right)$,

(2) $k$ is homogeneous of degree -1 and odd: for all $\lambda \neq 0, k(\lambda x, \lambda y)=$ $\lambda^{-1} k(x, y)$.

3. Let $k(x, y)$ be a double layer kernel and $0<\theta<2 \pi$. Define

$$
K_{\theta} f(t)=\int_{0}^{1} k(t-s \cos \theta,-s \sin \theta) f(s) d s .
$$

Then $K_{\theta}$ is a Hardy kernel operator with kernel

$$
k_{\theta}(t)=k(t-\cos \theta,-\sin \theta) .
$$

4. Let $k(x, y)$ be a double layer kernel. Then

$$
\lim _{y \rightarrow 0^{ \pm}} \int_{0}^{1} k(t-s, y) f(s) d s= \pm c_{k} f(t)+\pi k(1,0) H f(t),
$$

where

$$
c_{k}=\lim _{R \rightarrow \infty} \int_{-R}^{R} k(x, 1) d x=\lim _{\varepsilon \rightarrow 0^{+}} \int_{\varepsilon}^{\pi-\varepsilon} \frac{k(\cos \theta, \sin \theta)}{\sin \theta} d \theta .
$$


This is simply the observation that if we let

$$
\phi(t)=\left\{\begin{array}{l}
k(t, 1)-k(1,0) / t, \quad|t|>1, \\
k(t, 1), \quad|t|<1,
\end{array}\right.
$$

then $\phi(t)=O\left(1 / t^{2}\right)$ as $|t| \rightarrow \infty$, so that $\phi \in L^{1}(\mathbf{R})$. The function

$$
\frac{1}{y} \int_{0}^{1} \phi\left(\frac{t-s}{y}\right) f(s) d s
$$

is dominated by the Hardy-Littlewood maximal function of $f$ and approaches $\pm\left(\int \phi(x) d x\right) f$ in $L^{p}(J)$ (cf. Stein [St]). Since $k(x, 0)=k(1,0) / x$ is an odd function, $\left(\int \phi(x) d x\right)$ is given by $(2-8)$.

5. Let $k(x, y)$ be a double layer kernel. Let $\vec{\gamma}_{j}, j=1,2$, be two $C^{\infty}$ curves which intersect only at $(0,0)$. Assume that $d \vec{\gamma}_{j} /\left.d t\right|_{t=0}=\vec{u}_{j}$ are unit vectors, $\vec{u}_{1} \neq \vec{u}_{2}$, so that $\vec{\gamma}_{j}(t)=t \vec{u}_{j}+\vec{\varepsilon}_{j}(t)$, with $\vec{\varepsilon}_{j}(t)=O\left(t^{2}\right)$. Let

$$
K^{12} f(t)=\int_{0}^{1} k\left(\vec{\gamma}_{1}(t)-\vec{\gamma}_{2}(s)\right) f(s)\left|\frac{d \vec{\gamma}_{2}}{d s}\right| d s .
$$

Then $K^{12}$ is a Mellin operator whose principal symbol is the same as that of the Hardy kernel operator with kernel

$$
k^{12}(t)=k\left(t \vec{u}_{1}-\vec{u}_{2}\right) .
$$

To show this we assume $\vec{u}_{1}=(1,0)$ and $\vec{u}_{2}=(\cos \theta, \sin \theta), 0<\theta<2 \pi$. Then $k\left(\vec{\gamma}_{1}(t)-\vec{\gamma}_{2}(s)\right)=k(t-s \cos \theta,-s \sin \theta)+R(t, s)$, where

$$
R(t, s)=\int_{0}^{1} \vec{\varepsilon}(t, s) \cdot \nabla k((t-s \cos \theta,-s \sin \theta)+\tau \vec{\varepsilon}(t, s)) d \tau
$$

with $\vec{\varepsilon}(t, s)=\vec{\varepsilon}(t)-\vec{\varepsilon}(s)$. Since $\left|\vec{\gamma}_{1}(t)-\vec{\gamma}_{2}(s)\right| \approx t+s$, we can differentiate wrt $t$ to show that

$$
f(t) \mapsto \frac{d}{d t} \int_{0}^{1} R(t, s) f(s) d s
$$

can be dominated by a Hardy kernel operator. Hence $f(t) \mapsto \int_{0}^{1} R(t, s) f(s) d s$ is a compact operator on $L^{p}(J)$.

6. Let $\vec{\gamma}(t), 0 \leq t \leq 1$, be a $C^{\infty}$ curve and $k(x, y)$ a double layer kernel. Let

$$
K_{\vec{\gamma}} f(t)=\text { p.v. } \int_{0}^{1} k(\vec{\gamma}(t)-\vec{\gamma}(s)) f(s)\left|\frac{d \vec{\gamma}}{d s}\right| d s .
$$

Then $K_{\vec{\gamma}} \in \mathrm{Op} \Sigma_{0,1}(J)$ and has the same symbol as $\pi k\left(\vec{\gamma}^{\prime}(t)\right)|d \vec{\gamma} / d t| H$. Observe that if $\vec{\gamma}(t)-\vec{\gamma}(s)=\vec{\gamma}^{\prime}(t)(t)(t-s)+\vec{\varepsilon}(t, s)$, then

$$
k(\vec{\gamma}(t)-\vec{\gamma}(s))-\frac{k\left(\vec{\gamma}^{\prime}(t)\right)}{t-s}=\int_{0}^{1} \vec{\varepsilon}(t, s) \cdot \nabla k\left(\vec{\gamma}^{\prime}(t)(t-s)+\tau \vec{\varepsilon}(t, s)\right) d \tau,
$$

which gives rise to a compact operator on $L^{p}(J)$. 
7. In Example 6 assume that $\vec{\gamma}$ is smooth for $-1 \leq t \leq+1$ and $d \vec{\gamma}(0) / d t=$ $\vec{u}$. For $0 \leq t \leq 1$, let $\vec{\gamma}_{1}(t)=\vec{\gamma}(t), \vec{\gamma}_{2}(t)=\vec{\gamma}(-t)$. The operator $K^{12}$ of $(2-9)$ has the same symbol as the Hardy kernel $k(\vec{u}) \frac{1}{t+1}$. The kernel $s(t)=\frac{1}{\pi} \frac{1}{t+1}$ is the kernel for the Stieltjes transform and $\tilde{s}(z)=\csc \pi z$ [LP, (4.30)]. In particular, if we break a smooth curve $\vec{\gamma}(t),-1 \leq t \leq 1$ at $t=0$ the Hilbert transform p.v. $\int_{-1}^{+1} k(\vec{\gamma}(t)-\vec{\gamma}(s)) f(s)|d \vec{\gamma} / d s| d s$ is equivalent to the matrix of operators

$$
K=\left(\begin{array}{ll}
H_{\vec{\gamma}_{1}} & K^{12} \\
K^{21} & H_{\vec{\gamma}_{2}}
\end{array}\right),
$$

which has principal symbol at $t=0$ given by

$$
\pi k(\vec{u}) \times\left(\begin{array}{cc}
-\cot \pi z & \csc \pi z \\
-\csc \pi z & \cot \pi z
\end{array}\right)
$$

Note that the characteristic polynomial of the matrix in $(2-13)$ is $p(\lambda)=$ $(\lambda+i)(\lambda-i)$.

\section{LAYER POTENTIALS ON CURVILINEAR POLYGONS}

Let $\Omega^{+}$be a simply connected ${ }^{3}$ domain in $\mathbf{R}^{2}$ whose boundary is a simple closed curvilinear polygon. As $\partial \Omega^{+}$is traversed in the counterclockwise direction label the successive $N$ vertices as $P_{2}, P_{4}, \ldots, P_{2 N}=P_{0}$. Let $\vec{P}_{i} P_{j}$ be the oriented piece of $\partial \Omega^{+}$between $P_{i}$ and $P_{j}$. Suppose that $\overrightarrow{P_{2 k} P_{2 k+2}}$ is parametrized by $\vec{\gamma}(t), 0 \leq t \leq 2$. For $k=1, \ldots, N$, we introduce the false vertices $P_{2 k-1}=\vec{\gamma}_{2 k-2}(1)$ and then parametrize $\overrightarrow{P_{2 k} P_{2 k-1}}$ by $\vec{\gamma}_{2 k-1}(t) \equiv$ $\vec{\gamma}_{2 k-2}(2-t), 0 \leq t \leq 1$. When $t=0$ each parametrization is at one of the original vertices; if $t=1$, we are at a "midpoint". For $i=1, \ldots, 2 N$, let $\theta_{i}$ be the angle interior to $\Omega^{+}$at $P_{i}, 0<\theta_{i}<2 \pi$; of course $\theta_{2 k-1}=\pi$. We assume that at $t=0,1, d \vec{\gamma}_{j} / d t$ are unit vectors; the arclength on $\overrightarrow{P_{i} P_{i+1}}$ is given by $d \sigma=(-1)^{i}\left|d \vec{\gamma}_{i} / d t\right| d t$.

For $f$ a scalar or vector function in $L^{p}\left(\partial \Omega^{+}\right)$, we define $f^{i}(t)=f\left(\vec{\gamma}_{i}(t)\right)$, $0 \leq t \leq 1, i=1, \ldots, 2 N$.

Assume that $c(x, y)$ is scalar or matrix function such that for each $i, i=$ $1, \ldots, 2 N, c^{i}(t)=c\left(\vec{\gamma}_{i}(t)\right)$ is a smooth function. Let $k(x, y)$ be an odd double layer kernel. We define the double layer potential

$$
K f(P)=c(P) f(P)+\text { p.v. } \int_{\partial \Omega^{+}} k(P-Q) f(Q) d \sigma_{Q} .
$$

Let

$$
K^{i, j} f^{j}(t)=\delta_{i, j} c^{j}(t) f^{j}(t)+\text { p.v. } \int_{0}^{1} k\left(\vec{\gamma}_{i}(t)-\vec{\gamma}_{j}(s)\right) f^{j}(s)(-1)^{j}\left|\frac{d \vec{\gamma}_{j}}{d s}\right| d s,
$$

\footnotetext{
${ }^{3}$ If $\Omega^{+}$is multiply connected we apply the method to each component of $\partial \Omega^{+}$.
} 
so that

$$
(K f)^{i}(t)=\sum_{j=1}^{2 N} K^{i, j} f^{j}(t)
$$

we write $\mathbf{K}=\left(K^{i, j}\right)_{i, j=1, \ldots, 2 N}$ for the operator $K$ interpreted as a big system of Mellin operators on $L^{p}(J)$.

Except in the cases $j=i-1, i, i+1(\bmod 2 N)$, the operators $K^{i, j}$ have smooth kernels and thus are compact operators on $L^{p}(J)$. The operators $K^{2 k, 2 k-1}$ and $K^{2 k-1,2 k}$ are Hardy kernel operators whose symbol is calculated by $(2-7)$; in particular their principal symbol vanishes for $t>0$. The operators $K^{2 k, 2 k+1}$ and $K^{2 k+1,2 k}$ have principal symbol which vanishes for $0<t<1$; near $t=1$, to calculate $\operatorname{det}\left(\operatorname{Smbl}^{\frac{1}{p}}(\mathbf{K})\right)$, we can apply an even number of row and column transpositions to reduce the symbol matrix to $2 \times 2$ block diagonal form. After applying the reflection (1-3), we are again reduced to considering the previous case at $t=0$ with angle $\theta_{2 k+1}=\pi$. The determinants of the matrix of principal symbols are summarized in Theorem 2 .

Theorem 2. For $i=1, \ldots, 2 N,(\bmod 2 N)$, let $K^{(i)}$ denote the matrix of blocks

$$
K^{(i)}=\left(\begin{array}{cc}
K^{i-1, i-1} & K^{i-1, i} \\
K^{i, i-1} & K^{i, i}
\end{array}\right)
$$

Then at $t=0$,

$$
\operatorname{det}\left(\operatorname{Smbl}^{\frac{1}{p}}(\mathbf{K})\right)=\prod_{i=1}^{N} \operatorname{det}\left(\operatorname{Smbl}^{\frac{1}{p}}\left(K^{(2 i)}\right)\right) .
$$

At $t=1$,

$$
\operatorname{det}\left(\operatorname{Smbl}^{\frac{1}{p}}(\mathbf{K})\right)=\prod_{i=1}^{N} \operatorname{det}\left(\operatorname{Smbl}^{\frac{1}{p}}\left(K^{(2 i-1)}\right)\right) .
$$

At $z=\frac{1}{p} \pm i \infty$

$$
\operatorname{det}\left(\operatorname{Smbl}^{\frac{1}{p}}(\mathbf{K})\right)=\prod_{i=1}^{2 N} \operatorname{det}\left(\operatorname{Smbl}^{\frac{1}{p}}\left(K^{i, i}\right)\right) .
$$

\section{Elastostatic double layer potentials in a Plane Sector}

We give explicit calculations for the double layer potentials for the system of elastostatics and hydrostatics in a plane sector. In this section we fix $\theta$, $0<\theta<2 \pi$, and let $\Omega^{+}$be the sector of opening $\theta$ :

$$
\Omega^{+}=\{(x, y): x=r \cos \phi, y=r \sin \phi, 0<r<\infty, 0<\phi<\theta\} .
$$

Denote the two pieces of $\partial \Omega^{+}$as $S_{1}=\{(\tau, \rho): \tau>0, \rho=0\}$ and $S_{2}=\{(\tau, \rho): \tau=l \cos \theta, \rho=l \sin \theta, l>0\}$. We denote by $\vec{\nu}_{1}=-\mathbf{j}$ and 
$\vec{\nu}_{2}=-\sin \theta \mathbf{i}+\cos \theta \mathbf{j}$ the exterior normals to $\Omega^{+}$along $S_{1}$ and $S_{2}$. For a vector function $\mathbf{f} \in L^{p}\left(\partial \Omega^{+}\right)$, let $\mathbf{f}^{\mathbf{l}}(t)=\mathbf{f}(t, 0), \mathbf{f}^{2}(t)=\mathbf{f}(t \cos \theta, t \sin \theta)$.

For $(t, s) \notin \partial \Omega^{+}$, the double layer potential is defined as in (0-17):

$$
\begin{aligned}
\mathbf{u}_{\mathbf{T}}(t, s)= & \int_{\partial \Omega^{+}} \mathbf{T}_{\vec{\nu}(\tau, \rho)}(\boldsymbol{\Gamma}(t-\tau, s-\rho)) \mathbf{f}(\tau, \rho) d \sigma_{\tau, \rho} \\
= & \int_{0}^{\infty} \mathbf{T}_{\vec{\nu}_{1}}(\boldsymbol{\Gamma}(t-\tau, s)) \mathbf{f}^{1}(s) d \tau \\
& +\int_{0}^{\infty} \mathbf{T}_{\vec{\nu}_{2}}(\boldsymbol{\Gamma}(t-l \cos \theta, s-l \sin \theta)) \mathbf{f}^{2}(l)(-1) d l
\end{aligned}
$$

We have

$$
\lim _{s \rightarrow 0^{ \pm}} \mathbf{u}_{\mathbf{T}}(t, s)=\left(\mathbf{u}_{\mathbf{T}}^{ \pm}\right)^{1}(t)=\mathbf{K}_{\mathbf{T}}^{ \pm 11} \mathbf{f}^{1}(t)+\mathbf{K}_{\mathbf{T}}^{12} \mathbf{f}^{2}(t)
$$

where

$$
\begin{aligned}
\mathbf{K}_{\mathbf{T}}^{ \pm 11} \mathbf{f}^{1}(t) & = \pm \mathbf{I} \mathbf{f}^{1}(t)+\text { p.v. } \int_{0}^{\infty} \mathbf{T}_{\vec{\nu}_{1}(\tau, \rho)}(\boldsymbol{\Gamma}(t-\tau, 0)) \mathbf{f}^{1}(\tau) d \tau \\
\mathbf{K}_{\mathbf{T}}^{12} \mathbf{f}^{2}(t) & =-\int_{0}^{\infty} \mathbf{T}_{\vec{\nu}_{2}(\tau, \rho)}(\boldsymbol{\Gamma}(t-l \cos \theta, s-l \sin \theta)) \mathbf{f}^{2}(l) d l
\end{aligned}
$$

The singular integral operators in $\mathbf{K}_{\mathrm{T}}^{ \pm 11}$ are multiples of the Hilbert transform by (2-6) and the operator $\mathbf{K}_{\mathbf{T}}^{12}$ is a $2 \times 2$ matrix of Hardy kernel operators with $\operatorname{Smbl}^{\frac{1}{p}}\left(\mathbf{K}_{\mathbf{T}}^{12}\right)$ near $t=0$ given by the Mellin transform of the kernel. When the identity $\mathbf{I}$ and the Hilbert transform are considered as Mellin operators, their kernels are the distributions $\delta(t-1)$ and $h(t)=$ p.v. $\frac{1}{\pi} \frac{1}{t-1}$ respectively.

For $(t, 0) \in S_{1}$ and $(\cos \theta, \sin \theta) \in S_{2}$, we define

$$
d^{2}=t^{2}-2 t \cos \theta+1=(t-\cos \theta)^{2}+\sin ^{2} \theta .
$$

For $j=0,1,2,3$, let

$$
k_{j}(t)=\frac{1}{\pi} \frac{(t-\cos \theta)^{j}(\sin \theta)^{3-j}}{d^{4}} .
$$

Let $\mathscr{E}(x, y)$ be one of the scalar kernels in the matrix fundamental solution $(0-13)$. Then $k_{\mathscr{E}_{0}}=-\frac{\partial \mathscr{E}}{\partial y}$ and $k_{\mathscr{E}_{\tau}}=-\frac{\partial \mathscr{E}}{\partial x}$ are double layer kernels according to (Definition 2.1). We consider the following scalar double layer potentials:

$$
\begin{aligned}
& u_{\mathscr{E}}(t, s)=\int_{\partial \Omega^{+}} \frac{\partial}{\partial \rho}\{\mathscr{E}(t-\tau, s-\rho)\} f(\tau, \rho) d \sigma_{\tau, \rho}, \\
& u_{\mathscr{E}}(t, s)=\int_{\partial \Omega^{+}} \frac{\partial}{\partial \tau}\{\mathscr{E}(t-\tau, s-\rho)\} f(\tau, \rho) d \sigma_{\tau, \rho} .
\end{aligned}
$$

Taking limits as $s \rightarrow 0^{ \pm}$, we obtain the following Mellin operators on $L^{p}\left(\mathbf{R}^{+}\right)$: (4-10)

$$
\begin{gathered}
K_{\mathscr{E}_{\rho}}^{ \pm 11} f^{1}(t)=\lim _{s \rightarrow 0^{ \pm}} \int_{0}^{\infty}-\frac{\partial \mathscr{E}}{\partial y}(t-\tau, s) f^{1}(\tau) d \tau=\int_{0}^{\infty} k_{\mathscr{E}_{\rho}}^{ \pm 11}\left(\frac{t}{\tau}\right) f^{1}(\tau) \frac{d \tau}{\tau}, \\
K_{\mathscr{E}_{\rho}}^{12} f^{2}(t)=\int_{0}^{\infty}-\frac{\partial \mathscr{E}}{\partial y}(t-l \cos \theta,-l \sin \theta) f^{2}(l) d l=\int_{0}^{\infty} k_{\mathscr{E}_{\rho}}^{12}\left(\frac{t}{l}\right) f^{2}(l) \frac{d l}{l} .
\end{gathered}
$$


Similarly, we obtain the operators $K_{\mathscr{E}_{\tau}}^{ \pm 11}$ and $K_{\mathscr{E}_{\tau}}^{12}$ and their corresponding kernels $k_{\mathscr{E}_{\tau}}^{ \pm 11}$ and $k_{\mathscr{E}_{\tau}}^{12}$. The Mellin kernels obtained are given in the following kernel list.

(4-11)

$$
\begin{array}{lcccc}
\mathscr{E}(t-\tau, s-\rho) & k_{\mathscr{E}_{\rho}}^{ \pm 11} & k_{\mathscr{E}_{\tau}}^{ \pm 11} & k_{\mathscr{E}_{\rho}}^{12} & k_{\mathscr{E}_{\tau}}^{12} \\
\frac{1}{2 \pi} \log \left((\tau-t)^{2}+(\rho-s)^{2}\right) & \mp \delta & -h & k_{0}+k_{2} & -k_{1}-k_{3} \\
\frac{1}{\pi} \frac{(\tau-t)(\rho-s)}{(\tau-t)^{2}+(\rho-s)^{2}} & -h & 0 & k_{1}-k_{3} & k_{0}-k_{2} \\
\frac{1}{\pi} \frac{(\tau-t)^{2}}{(\tau-t)^{2}+(\rho-s)^{2}} & \pm \delta & 0 & -2 k_{2} & -2 k_{1} \\
\frac{1}{\pi} \frac{(\rho-s)^{2}}{(\tau-t)^{2}+(\rho-s)^{2}} & \mp \delta & 0 & 2 k_{2} & 2 k_{1}
\end{array}
$$

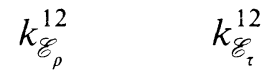

In (4-11) we have used the notation $\delta$ and $h$ for the distribution Mellin kernels $\delta(t-1)$ and p.v. $\frac{1}{\pi} \frac{1}{t-1}$ respectively.

To show the explicit dependence of the kernels on the parameter $b=\frac{\lambda+\mu}{\lambda+2 \mu}$ (cf. (0-3)), we note the following "tricks" which follow from $(0-3)$ and $(0-14)$ : (4-12)

$$
\begin{aligned}
& \mu m=\frac{b}{2}, \quad \mu n=1-\frac{b}{2}, \quad \mu(n+2 m)=1+\frac{b}{2}, \quad \lambda(m-n)=1-2 b, \\
& \mu(2 m-n)=\frac{3}{2} b-1, \quad \mu(n-m)=1-b, \quad \mu(n+m)=1 .
\end{aligned}
$$

We now give the structure of the operators $\mathbf{K}_{\mathbf{T}}^{ \pm 11}$ and $\mathbf{K}_{\mathbf{N}}^{ \pm 11}$.

Theorem 3. Let

$$
\mathbf{K}_{\mathbf{T}^{0}}^{11}=\left(\begin{array}{cc}
0 & H \\
-H & 0
\end{array}\right)
$$

Then

$$
\begin{aligned}
& \mathbf{K}_{\mathbf{T}}^{ \pm 11}= \pm \mathbf{I}+(1-b) \mathbf{K}_{\mathbf{T}^{0}}^{11}, \\
& \mathbf{K}_{\mathbf{N}}^{ \pm 11}= \pm \mathbf{I}+\frac{b}{2} \mathbf{K}_{\mathbf{T}^{0}}^{11}
\end{aligned}
$$

Proof. With $\vec{\nu}=-\mathbf{j}$, we have that

$$
\mathbf{T}_{\vec{\nu}}(\mathbf{u}(\tau, \rho))=-\left(\begin{array}{c}
\mu u_{1, \rho} \\
\lambda u_{1, \tau}+(\lambda+2 \mu) u_{2, \rho}
\end{array}\right) .
$$

We apply $\mathbf{T}_{\vec{\nu}(\tau, \rho)}$ to the columns of the fundamental matrix $\boldsymbol{\Gamma}(t-\tau, s-\rho)$ and take limits as $s \rightarrow 0^{ \pm}$. As a sample calculation we calculate the kernel in 
the 2,1 position. Using the kernel list (4-11), we obtain

$$
\begin{aligned}
-k_{\mathbf{T}, 21}^{ \pm 11} & =\lambda[n(-h)-m \cdot 0]+(\lambda+2 \mu)[-m(-h)] \\
& =-h[\lambda n+(\lambda+2 \mu)(-m)] \\
& =-h[\lambda(n-m)-2 \mu m] \\
& =-h\left[2 b-1-2 \frac{b}{2}\right] \\
& =(1-b) h .
\end{aligned}
$$

Similarly

$$
-k_{\mathbf{N}, 21}^{ \pm 11}=(\lambda+\mu)[n(-h)-m \cdot 0]+(\lambda+2 \mu)[-m(-h)]
$$

The method of simplification to be consistently applied is to collect the coefficients of $\lambda$ and $\mu$ and then to use the tricks (4-12) to write the coefficients in terms of $b$.

The remaining very tedious calculations are left to the reader.

To calculate the kernels in $\mathbf{K}_{\mathbf{T}}^{12}$ and $\mathbf{K}_{\mathbf{N}}^{12}$, we split the operators into

$$
\mathbf{K}_{\mathbf{T}}^{12}=\sin \theta \mathbf{K}_{\mathbf{T}_{\mathbf{i}}}-\cos \theta \mathbf{K}_{\mathbf{T}_{\mathbf{j}}}
$$

where

$$
\begin{aligned}
& \mathbf{K}_{\mathbf{T}_{\mathbf{i}}}^{12} \mathbf{f}^{2}(t)=\int_{0}^{\infty} \mathbf{T}_{\mathbf{i}}(\boldsymbol{\Gamma}(t-l \cos \theta,-l \sin \theta)) \mathbf{f}^{2}(l) d l \\
& \mathbf{K}_{\mathbf{T}_{\mathbf{j}}}^{12} \mathbf{f}^{2}(t)=\int_{0}^{\infty} \mathbf{T}_{\mathbf{j}}(\boldsymbol{\Gamma}(t-l \cos \theta,-l \sin \theta)) \mathbf{f}^{2}(l) d l
\end{aligned}
$$

and

$$
\begin{aligned}
& \mathbf{K}_{\mathbf{N}_{\mathbf{i}}}^{12} \mathbf{f}^{2}(t)=\int_{0}^{\infty} \mathbf{N}_{\mathbf{i}}(\boldsymbol{\Gamma}(t-l \cos \theta,-l \sin \theta)) \mathbf{f}^{2}(l) d l, \\
& \mathbf{K}_{\mathbf{N}_{\mathbf{j}}}^{12} \mathbf{f}^{2}(t)=\int_{0}^{\infty} \mathbf{N}_{\mathbf{j}}(\boldsymbol{\Gamma}(t-l \cos \theta,-l \sin \theta)) \mathbf{f}^{2}(l) d l .
\end{aligned}
$$

Note that the $(-1)$ from the orientation has been omitted in the definitions (4-18) and (4-19).

Theorem 4. The operators in (4-18) and (4-19) have the following structure:

$$
\begin{array}{ll}
\mathbf{K}_{\mathbf{T}_{\mathbf{i}}}^{12}=\mathbf{K}_{\mathbf{T}_{\mathbf{i}}^{0}}^{12}+b \mathbf{K}_{\mathbf{i}^{b}}, & \mathbf{K}_{\mathbf{T}_{\mathbf{j}}}^{12}=\mathbf{K}_{\mathbf{T}_{\mathbf{j}}^{0}}^{12}+b \mathbf{K}_{\mathbf{j}^{b}}, \\
\mathbf{K}_{\mathbf{N}_{\mathbf{i}}}^{12}=\mathbf{K}_{\mathbf{N}_{\mathbf{i}}^{0}}^{12}+\frac{b}{2} \mathbf{K}_{\mathbf{i}^{b}}, & \mathbf{K}_{\mathbf{N}_{\mathbf{i}}}^{12}=\mathbf{K}_{\mathbf{N}_{\mathbf{j}}^{0}}^{12}+\frac{b}{2} \mathbf{K}_{\mathbf{j}^{b}},
\end{array}
$$


where the Hardy kernels are

$$
\begin{aligned}
\mathbf{K}_{\mathbf{T}_{\mathbf{i}}^{0}}^{12} & =\left(\begin{array}{cc}
-k_{1}-k_{3} & -k_{0}-k_{2} \\
k_{0}+k_{2} & -k_{1}-k_{3}
\end{array}\right), \\
\mathbf{K}_{\mathbf{i}^{b}}^{12} & =\left(\begin{array}{cc}
k_{1}-k_{3} & k_{0}+3 k_{2} \\
-k_{0}+k_{2} & -k_{1}+k_{3}
\end{array}\right), \\
\mathbf{K}_{\mathbf{N}_{\mathbf{i}}^{0}}^{12} & =\left(\begin{array}{cc}
-k_{1}-k_{3} & 0 \\
0 & -k_{1}-k_{3}
\end{array}\right), \\
\mathbf{K}_{\mathbf{T}_{\mathbf{j}}^{0}}^{12} & =\left(\begin{array}{cc}
k_{0}+k_{2} & -k_{1}-k_{3} \\
k_{1}+k_{3} & k_{0}+k_{2}
\end{array}\right), \\
\mathbf{K}_{\mathbf{j}^{b}}^{12} & =\left(\begin{array}{cc}
-k_{0}+k_{2} & -k_{1}+k_{3} \\
-3 k_{1}-k_{3} & k_{0}-k_{2}
\end{array}\right), \\
\mathbf{K}_{\mathbf{N}_{\mathbf{j}}^{12}}^{12} & =\left(\begin{array}{cc}
k_{0}+k_{2} & 0 \\
0 & k_{0}+k_{2}
\end{array}\right) .
\end{aligned}
$$

Proof. A typical computation is for the kernel in the 1,1 position.

$$
\begin{aligned}
k_{\mathbf{T}_{\mathbf{i}}, 11}^{12} & =(\lambda+2 \mu)\left[n\left(-k_{1}-k_{3}\right)-m\left(-2 k_{1}\right)\right]+\lambda(-m)\left(k_{1}-k_{3}\right) \\
& =k_{1}[(\lambda+2 \mu)(-n+2 m)-\lambda m]+k_{3}[(\lambda+2 \mu)(-n)+\lambda m] .
\end{aligned}
$$

To simplify the coefficients of $k_{1}$ and $k_{3}$, collect the coefficients of $\lambda$ and $\mu$, and apply the tricks (4-12) to obtain

$$
k_{\mathbf{T}_{\mathbf{i}}, 11}^{12}=k_{1}(-1+b)+k_{3}(-1-b) \text {. }
$$

In calculating the remaining kernels, note that the coefficients to be calculated for $k_{\mathbf{T}_{\mathbf{j}}, r s}^{12}$ are the negatives of the coefficients calculated for $k_{\mathbf{T}_{\mathbf{i}}, s r}^{12}$.

Again the very tedious details are left to the reader.

Taking into account the $(-1)$ introduced by the orientation of the ray $S_{2}$, we have

$$
\begin{aligned}
& \mathbf{K}_{\mathbf{T}}^{12}=\sin \theta \mathbf{K}_{\mathbf{T}_{\mathbf{i}}}^{12}-\cos \theta \mathbf{K}_{\mathbf{T}_{\mathbf{j}}}^{12}, \\
& \mathbf{K}_{\mathbf{N}}^{12}=\sin \theta \mathbf{K}_{\mathbf{N}_{\mathbf{i}}}^{12}-\cos \theta \mathbf{K}_{\mathbf{N}_{\mathbf{j}}}^{12}
\end{aligned}
$$

We introduce

$$
\begin{aligned}
& \mathbf{K}_{\mathbf{T}^{0}}^{12}=\sin \theta \mathbf{K}_{\mathbf{T}_{\mathbf{i}^{0}}}^{12}-\cos \theta \mathbf{K}_{\mathbf{T}_{\mathbf{j}^{0}}}^{12}, \\
& \mathbf{K}_{\mathbf{N}^{0}}^{12}=\sin \theta \mathbf{K}_{\mathbf{N}_{\mathbf{0}^{0}}}^{12}-\cos \theta \mathbf{K}_{\mathbf{N}_{\mathbf{j}^{0}}}^{12}, \\
& \mathbf{K}_{\vec{\nu}^{b}}^{12}=\sin \theta \mathbf{K}_{\mathbf{i}^{b}}^{12}-\cos \theta \mathbf{K}_{\mathbf{j}^{b}}^{12},
\end{aligned}
$$

so that

$$
\begin{aligned}
& \mathbf{K}_{\mathbf{T}}^{12}=\mathbf{K}_{\mathbf{T}^{0}}^{12}+b \mathbf{K}_{\vec{\nu}^{b}}^{12}, \\
& \mathbf{K}_{\mathbf{N}}^{12}=\mathbf{K}_{\mathbf{N}^{0}}^{12}+\frac{b}{2} \mathbf{K}_{\vec{\nu}^{b}}^{12} .
\end{aligned}
$$

Next we calculate $\mathbf{K}_{\{\cdot\}}^{21}$ and $\mathbf{K}_{\{\cdot\}}^{22}$. 
Let $U$ be the reflection about the ray $\left\{(t, s)=\left(l \cos \frac{\theta}{2}, l \sin \frac{\theta}{2}\right): l>0\right\}$ :

$$
U=\left(\begin{array}{cc}
\cos \theta & \sin \theta \\
\sin \theta & -\cos \theta
\end{array}\right) .
$$

Note that $U U=\mathbf{I}_{2}$ and that $\operatorname{det} U=-1$.

Then it is "obvious" geometrically or may be verified by a calculation that

$$
\begin{array}{ll}
\mathbf{K}_{\mathbf{T}}^{21}=U \mathbf{K}_{\mathbf{T}}^{12} U, & \mathbf{K}_{\mathbf{T}}^{ \pm 22}=U \mathbf{K}_{\mathbf{T}}^{ \pm 11} U, \\
\mathbf{K}_{\mathbf{N}}^{21}=U \mathbf{K}_{\mathbf{N}}^{12} U, & \mathbf{K}_{\mathbf{N}}^{ \pm 22}=U \mathbf{K}_{\mathbf{N}}^{ \pm 11} U .
\end{array}
$$

Hence both $\mathbf{K}_{\mathbf{T}}^{ \pm}$and $\mathbf{K}_{\mathbf{N}}^{ \pm}$have the structure

$$
\mathbf{K}_{\{\cdot\}}^{ \pm}=\left(\begin{array}{cc}
\mathbf{K}_{\{\cdot\}}^{ \pm 11} & \mathbf{K}_{\{\cdot\}}^{12} \\
U \mathbf{K}_{\{\cdot\}}^{12} U & U \mathbf{K}_{\{\cdot\}}^{ \pm 11} U
\end{array}\right) .
$$

We let $\hat{U}$ be the $4 \times 4$ matrix

$$
\hat{U}=\left(\begin{array}{cc}
\mathbf{I}_{2} & 0 \\
0 & U
\end{array}\right) .
$$

Then

$$
\hat{U} \mathbf{K}_{\{\cdot\}}^{ \pm} \hat{U}=\left(\begin{array}{cc}
\mathbf{K}_{\cdot \cdot\}}^{ \pm 11} & \mathbf{K}_{\{\cdot\}}^{12} U \\
\mathbf{K}_{\{\cdot\}}^{12} U & \mathbf{K}_{\{\cdot\}}^{ \pm 11}
\end{array}\right) .
$$

\section{THE SYMBOLS IN A PLANE SECTOR}

We are now reduced to calculating the determinant of a matrix of Mellin symbols of the form

$$
\operatorname{Smbl}^{\frac{1}{p}}\left(\hat{U} \mathbf{K}_{\{\cdot\}}^{ \pm} \hat{U}\right)=\left(\begin{array}{cc}
\tilde{\mathbf{K}}_{\{\cdot \cdot}^{ \pm 11} & \tilde{\mathbf{K}}_{\{\cdot \cdot\}}^{12} U \\
\tilde{\mathbf{K}}_{\{\cdot\}}^{12} U & \tilde{\mathbf{K}}_{\{\cdot\}}^{ \pm 11}
\end{array}\right) .
$$

First we note that if $A$ and $B$ are $2 \times 2$ matrices, then

$$
\operatorname{det}\left(\begin{array}{ll}
A & B \\
B & A
\end{array}\right)=\operatorname{det}(A-B) \cdot \operatorname{det}(A+B) \text {. }
$$

Our goal is to express $\operatorname{det}\left(\tilde{\mathbf{K}}_{\{\cdot\}}^{ \pm 11} \pm \tilde{\mathbf{K}}_{\{\cdot\}}^{12} U\right)$ as the difference of two squares so that the zeroes can easily be found.

We shall call antireflective a matrix of the form $C=\left(\begin{array}{cc}c_{11} & c_{12} \\ -c_{12} & c_{11}\end{array}\right)$; note that $\operatorname{det} C=c_{11}^{2}+c_{12}^{2}$. We shall call reflective a matrix of the form $D=\left(\begin{array}{cc}d_{11} & d_{12} \\ d_{12} & -d_{11}\end{array}\right)$; note that $\operatorname{det} D=-\left(d_{11}^{2}+d_{12}^{2}\right)$. Finally observe that if $C$ is antireflective and $D$ is reflective, then

$$
\operatorname{det}(C \pm D)=\left(c_{11}^{2}+c_{12}^{2}\right)-\left(d_{11}^{2}+d_{12}^{2}\right)=\operatorname{det} C+\operatorname{det} D .
$$

First we record the structure of $\operatorname{Smbl}^{\frac{1}{p}}\left(\mathbf{K}_{\{\cdot\}}^{ \pm 11}\right)$ near $t=0$. If $\mathbf{K}_{\mathbf{T}^{0}}^{11}$ is as defined in (4-13), it is immediate that near $t=0$,

$$
\sin \pi z \operatorname{Smbl}^{\frac{1}{p}}\left(\mathbf{K}_{\mathbf{T}^{0}}^{11}\right)(t, z)=\left(\begin{array}{cc}
0 & -\cos \pi z \\
\cos \pi z & 0
\end{array}\right) ;
$$

the matrix in (5-4) is antireflective. 
Theorem 5. Near $t=0$, the matrices $\operatorname{Smbl}^{\frac{1}{p}}\left(\mathbf{K}_{\{\cdot\}}^{ \pm 11}\right)$ are antireflective; the symbols are given by

$$
\begin{aligned}
& \sin \pi z \operatorname{Smbl}^{\frac{1}{p}}\left(\mathbf{K}_{\mathrm{T}}^{ \pm 11}\right)(t, z)=\left(\begin{array}{cc} 
\pm \sin \pi z & -(1-b) \cos \pi z \\
(1-b) \cos \pi z & \pm \sin \pi z
\end{array}\right), \\
& \sin \pi z \operatorname{Smbl}^{\frac{1}{p}}\left(\mathbf{K}_{\mathbf{N}}^{ \pm 11}\right)(t, z)=\left(\begin{array}{cc} 
\pm \sin \pi z & -\frac{b}{2} \cos \pi z \\
\frac{b}{2} \cos \pi z & \pm \sin \pi z
\end{array}\right) .
\end{aligned}
$$

To calculate the symbols of the Hardy kernel operators in (4-21), we give the Mellin transforms of the kernels. First we introduce

$$
\begin{aligned}
& C_{\theta}(z)=\cos ((\pi-\theta) z+\theta), \\
& S_{\theta}(z)=\sin ((\pi-\theta) z+\theta) .
\end{aligned}
$$

We list the following table of Mellin tranforms for the kernels $k_{j}(t)$ defined by $(4-6)$ :

$$
\begin{aligned}
& \sin \pi z \tilde{k}_{0}(z)=\frac{1}{2}\left\{(-z+2) \sin \theta C_{\theta}(z-1)-\cos \theta S_{\theta}(z-1)\right\}, \\
& \sin \pi z \tilde{k}_{1}(z)=-\frac{1}{2}\left\{(z-1) \sin \theta S_{\theta}(z-1)\right\}, \\
& \sin \pi z \tilde{k}_{2}(z)=\frac{1}{2}\left\{z \sin \theta C_{\theta}(z-1)-\cos \theta S_{\theta}(z-1)\right\}, \\
& \sin \pi z \tilde{k}_{3}(z)=\frac{1}{2}\left\{(z+1) \sin \theta S_{\theta}(z-1)+2 \cos \theta C_{\theta}(z-1)\right\} .
\end{aligned}
$$

For obvious reasons we note the following formulas which follow easily from (5-7) and the trigonometric addition formulas.

$$
\begin{aligned}
\sin \pi z\left(\tilde{k}_{0}(z)-\tilde{k}_{2}(z)\right) & =(-z+1) \sin \theta C_{\theta}(z-1), \\
\sin \pi z\left(\tilde{k}_{1}(z)-\tilde{k}_{3}(z)\right) & =-z \sin \theta S_{\theta}(z-1)-\cos \theta C_{\theta}(z-1), \\
\sin \pi z\left(\tilde{k}_{0}(z)+3 \tilde{k}_{2}(z)\right) & =(z+1) \sin \theta C_{\theta}(z-1)-2 \cos \theta S_{\theta}(z-1), \\
\sin \pi z\left(3 \tilde{k}_{1}(z)-\tilde{k}_{3}(z)\right) & =(-z+2) \sin \theta S_{\theta}(z-1)+\cos \theta C_{\theta}(z-1), \\
\sin \pi z\left(\tilde{k}_{0}(z)+\tilde{k}_{2}(z)\right) & =\sin \theta C_{\theta}(z-1)-\cos \theta C_{\theta}(z-1) \\
& =-\sin ((\pi-\theta)(z-1)), \\
\sin \pi z\left(\tilde{k}_{1}(z)+\tilde{k}_{3}(z)\right) & =\cos \theta C_{\theta}(z-1)+\sin \theta S_{\theta}(z-1) \\
& =\cos ((\pi-\theta)(z-1)) .
\end{aligned}
$$

The structure of the symbols of the operators (4-24) is explained in Theorem 6.

We first introduce the reflective matrix

$$
V=\left(\begin{array}{cc}
\sin \theta & -\cos \theta \\
-\cos \theta & -\sin \theta
\end{array}\right) .
$$

Theorem 6. The symbols of the operators $\mathbf{K}_{\mathbf{T}^{0}}^{12} U$ and $\mathbf{K}_{\mathbf{N}^{0}}^{12} U$ are reflective matrices and satisfy

$$
\begin{aligned}
\sin \pi z \operatorname{Smbl}^{\frac{1}{p}}\left(\mathbf{K}_{\mathbf{T}^{0}}^{12} U\right)(t, z) & =\left(\begin{array}{cc}
\sin (\pi-\theta)(z-1) & -\cos (\pi-\theta)(z-1) \\
-\cos (\pi-\theta)(z-1) & -\sin (\pi-\theta)(z-1)
\end{array}\right), \\
& =-\sin (\pi-\theta) z U-\cos (\pi-\theta) z V
\end{aligned}
$$

$\sin \pi z \operatorname{Smbl}^{\frac{1}{p}}\left(\mathbf{K}_{\mathbf{N}^{0}}^{12} U\right)(t, z)=-\sin (\pi-\theta) z U$. 
The symbol of the operator $\mathbf{K}_{\vec{\nu}^{b}}^{12}$ is a matrix of the form $\{z \times$ antireflective + reflective $\}$ and satisfies

$$
\begin{aligned}
\sin \pi z \operatorname{Smbl}^{\frac{1}{p}}\left(\mathbf{K}_{\vec{\nu}^{b}}^{12} U\right)(t, z)= & z \sin \theta\left(\begin{array}{cc}
\cos (\pi-\theta) z & -\sin (\pi-\theta) z \\
\sin (\pi-\theta) z & \cos (\pi-\theta) z
\end{array}\right) \\
& +\cos (\pi-\theta) z V .
\end{aligned}
$$

Finally we are ready to calculate $\operatorname{det}\left(\tilde{\mathbf{K}}_{\{\cdot\}}^{ \pm 11} \pm \tilde{\mathbf{K}}_{\{\cdot\}}^{12} U\right)$. To avoid further confusion, we now calculate $\operatorname{det} \operatorname{Smbl}^{\frac{1}{p}}\left(\mathbf{K}_{\{\cdot\}}^{+}\right)$.

Define

$$
\begin{aligned}
& f_{\mathbf{T}}^{\oplus \pm}(z)=\operatorname{det}\left(\sin \pi z\left(\tilde{\mathbf{K}}_{\mathbf{T}}^{+11} \pm \tilde{\mathbf{K}}_{\mathbf{T}}^{12} U\right)\right), \\
& f_{\mathbf{N}}^{\oplus \pm}(z)=\operatorname{det}\left(\sin \pi z\left(\tilde{\mathbf{K}}_{\mathbf{N}}^{+11} \pm \tilde{\mathbf{K}}_{\mathbf{N}}^{12} U\right)\right) .
\end{aligned}
$$

Next define

$$
\begin{aligned}
g_{\mathbf{T}}^{++}(z) & =b z \sin \theta+(2-b) \sin (2 \pi-\theta) z \\
& =-b z \sin (2 \pi-\theta)+(2-b) \sin (2 \pi-\theta) z, \\
g_{\mathbf{T}}^{--}(z) & =b z \sin \theta-(2-b) \sin (2 \pi-\theta) z \\
& =-b z \sin (2 \pi-\theta)-(2-b) \sin (2 \pi-\theta) z, \\
g_{\mathbf{T}}^{+-} & =b(z \sin \theta+\sin \theta z), \\
g_{\mathbf{T}}^{-+} & =b(z \sin \theta-\sin \theta z) .
\end{aligned}
$$

Let

$$
\begin{aligned}
g_{\mathbf{N}}^{++}(z) & =\frac{b}{2} z \sin \theta+\left(1-\frac{b}{2}\right) \sin (2 \pi-\theta) z \\
& =-\frac{b}{2} z \sin (2 \pi-\theta)+\left(1-\frac{b}{2}\right) \sin (2 \pi-\theta) z, \\
g_{\mathbf{N}}^{--}(z) & =\frac{b}{2} z \sin \theta-\left(1-\frac{b}{2}\right) \sin (2 \pi-\theta) z \\
& =-\frac{b}{2} z \sin \theta-\left(1-\frac{b}{2}\right) \sin (2 \pi-\theta) z, \\
g_{\mathbf{N}}^{+-} & =\frac{b}{2} z \sin \theta+\left(1+\frac{b}{2}\right) \sin \theta z, \\
g_{\mathbf{N}}^{-+} & \left.=\frac{b}{2} z \sin \theta-\left(1+\frac{b}{2}\right) \sin \theta z\right) .
\end{aligned}
$$

Theorem 7. We have that

$$
\begin{aligned}
& f_{\mathbf{T}}^{\oplus \pm}(z)=g_{\mathbf{T}}^{ \pm+}(z) \cdot g_{\mathbf{T}}^{ \pm-}(z), \\
& f_{\mathbf{N}}^{\oplus \pm}(z)=g_{\mathbf{N}}^{ \pm+}(z) \cdot g_{\mathbf{N}}^{ \pm-}(z) .
\end{aligned}
$$

Proof. Let

$$
A^{ \pm}=\sin \pi z\left(\tilde{\mathbf{K}}_{\mathrm{T}}^{+11} \pm \tilde{\mathbf{K}}_{\mathrm{T}}^{12} U\right)
$$


Using (4-25), (5-10), and (5-11), the antireflective part of $A^{ \pm}$is $(5-17)$

$$
A_{\text {anti }}^{ \pm}=\sin \pi z\left(\mathbf{I}_{2}+(1-b) \tilde{\mathbf{K}}_{\mathrm{T}^{0}}^{11}\right) \pm z(\sin \theta)\left(\begin{array}{cc}
\cos (\pi-\theta) z & -\sin (\pi-\theta) z \\
\sin (\pi-\theta) z & \cos (\pi-\theta) z
\end{array}\right),
$$

which has determinant given by

$(5-18)(\sin \pi z \pm b z \sin \theta \cos (\pi-\theta) z)^{2}+((1-b) \cos \pi z \pm b z \sin \theta \sin (\pi-\theta) z)^{2}$.

From (4-25) and (5-11), the reflective part of $A^{ \pm}$is

$$
A_{\text {refl }}^{ \pm}= \pm\left(\tilde{\mathbf{K}}_{\mathrm{T}^{0}}^{12} U+b \cos (\pi-\theta) z V\right),
$$

which has determinant given by

$$
\begin{gathered}
-\left[(\cos \theta \sin (\pi-\theta) z+(1-b) \sin \theta \cos (\pi-\theta) z)^{2}\right. \\
\left.+(\sin \theta \sin (\pi-\theta) z-(1-b) \cos \theta \cos (\pi-\theta) z)^{2}\right] \\
=-\left[\sin ^{2}(\pi-\theta) z+(1-b)^{2} \cos ^{2}(\pi-\theta) z\right]
\end{gathered}
$$

Thus

$$
\begin{aligned}
f_{\mathbf{T}}^{\oplus \pm}(z)=\{ & \left.\sin ^{2} \pi z-\sin ^{2}(\pi-\theta) z\right\}+(1-b)^{2}\left\{\cos ^{2} \pi z-\cos ^{2}(\pi-\theta) z\right\} \\
& +b^{2} z^{2} \sin ^{2} \theta \pm 2 b z \sin \theta\{\sin \pi z \cos (\pi-\theta) z \\
& +(1-b) \cos \pi z \sin (\pi-\theta) z\} .
\end{aligned}
$$

In the last two terms of $(5-21)$ we complete the square to obtain $(5-22)$

$f_{\mathbf{T}}^{\oplus \pm}(z)=(b z \sin \theta \pm(\sin \pi z \cos (\pi-\theta) z+(1-b) \cos \pi z \sin (\pi-\theta) z))^{2}+$ rest,

where

$$
\begin{aligned}
\text { rest }= & \sin ^{2} \pi z-\sin ^{2}(\pi-\theta) z+(1-b)^{2}\left[\cos ^{2} \pi z-\cos ^{2}(\pi-\theta) z\right] \\
& -(\sin \pi z \cos (\pi-\theta) z+(1-b) \cos \pi z \sin (\pi-\theta) z)^{2} \\
= & -2(1-b) \sin \pi z \cos (\pi-\theta) z \cos \pi z \sin (\pi-\theta) z \\
& +\left\{\sin ^{2} \pi z-\sin ^{2}(\pi-\theta) z-\sin ^{2} \pi z \cos ^{2}(\pi-\theta) z\right\} \\
& +(1-b)^{2}\left\{\cos ^{2} \pi z-\cos ^{2}(\pi-\theta) z-\cos ^{2} \pi z \sin ^{2}(\pi-\theta) z\right\} .
\end{aligned}
$$

The two terms in $\{\cdot\}$ simplify respectively to $-\cos ^{2} \pi z \sin ^{2}(\pi-\theta) z$ and $-\sin ^{2} \pi z \cos ^{2}(\pi-\theta) z$ so that

$$
\text { rest }=-\{\cos \pi z \sin (\pi-\theta) z+(1-b) \sin \pi z \cos (\pi-\theta) z\}^{2} .
$$

From (5-22) and (5-24), the function $f_{\mathbf{T}}^{\oplus \pm}$ has been written as the difference of two squares $\alpha^{2}-\beta^{2}$ so that of course $f_{\mathbf{T}}^{\oplus \pm}=(\alpha+\beta)(\alpha-\beta)$. That the terms have the form given by (5-15) follows from the addition formulas.

The explicit calculations for $f_{\mathbf{N}}^{\oplus \pm}$ proceed in a like manner. 
Remark. In a similar manner we may calculate

$$
\begin{aligned}
& f_{\mathbf{T}}^{\ominus \pm}(z)=\operatorname{det}\left(\sin \pi z\left(\tilde{\mathbf{K}}_{\mathbf{T}}^{-11} \pm \tilde{\mathbf{K}}_{\mathbf{T}}^{12} U\right)\right), \\
& f_{\mathbf{N}}^{\ominus \pm}(z)=\operatorname{det}\left(\sin \pi z\left(\tilde{\mathbf{K}}_{\mathbf{N}}^{-11} \pm \tilde{\mathbf{K}}_{\mathbf{N}}^{12} U\right)\right) .
\end{aligned}
$$

In the calculation the determinant of the reflective part is unchanged and for the determinant of the antireflective part (5-18) is replaced by

$(5-26)(-\sin \pi z \pm b z \sin \theta \cos (\pi-\theta) z)^{2}+((1-b) \cos \pi z \pm b z \sin \theta \sin (\pi-\theta) z)^{2}$.

The final result is that

$(5-27)$

$\operatorname{det}\left(\sin \pi z \operatorname{Smbl}^{\frac{1}{p}}\left(\mathbf{K}_{\mathbf{T}}^{-}\right)\right)=(b z \sin \theta-b \sin (2 \pi-\theta z))(b z \sin \theta-(2-b) \sin \theta z)$

$$
\times(b z \sin \theta+b \sin (2 \pi-\theta z))(b z \sin \theta+(2-b) \sin \theta z) .
$$

As expected, $\operatorname{det}\left(\sin \pi z \operatorname{Smbl}^{\frac{1}{p}}\left(\mathbf{K}_{\mathbf{T}}^{-}\right)\right)$has the same form as

$$
\operatorname{det}\left(\sin \pi z \operatorname{Smbl}^{\frac{1}{p}}\left(\mathbf{K}_{\mathbf{T}}^{+}\right)\right),
$$

with the roles of $\theta$ and $2 \pi-\theta$ interchanged, since $2 \pi-\theta$ is the "interior" angle for the complement of $\Omega^{+}$.

\section{THE SINGULARITIES OF THE PRINCIPAL SYMBOL}

The zeroes and change in argument of $\operatorname{det}\left(\operatorname{Smbl}^{\frac{1}{p}}\left(\mathbf{K}_{\{\cdot\}}^{+}\right)\right)=(\sin \pi z)^{-4} f_{\{\cdot\}}^{\oplus+}(z)$. $f_{\{\cdot\}}^{\oplus-}(z)$ can be easily calculated from $(5-15)$. Essentially we must consider functions of the form

$$
g_{\alpha, \gamma}(z)=\frac{\sin \gamma z}{\gamma z}-\alpha \frac{\sin \gamma}{\gamma}
$$

where $-1 \leq \alpha \leq 1$ and $0<\gamma<2 \pi$. An interesting discussion of all the complex zeroes of $(6-1)$ is given in Vasilopoulos [V] or Karal and Karp [KK]. Let $g(Z)=\sin Z / Z$; of course $g(Z)$ has simple zeroes at $Z= \pm n \pi, n=$ $1,2, \ldots$ The next lemma is a summary of the remarks of $[\mathrm{V}, \mathrm{pp} .57 \mathrm{ff}$. $]$ and is proved using the Argument Principle.

Lemma 6.1. Let $0<C<1$. Then the equation

$$
g(Z)-C=0
$$

has exactly one root in the strip $\Gamma_{0, \pi}$, has no roots in the strips $\Gamma_{(2 n-1) \pi, 2 n \pi}, n=$ $1,2, \ldots$, and has exactly two roots in the strips $\Gamma_{2 n \pi,(2 n+1) \pi}, n=1,2, \ldots$.

The equation

$$
g(Z)+C=0
$$

has no roots in the strips $\Gamma_{(2 n-2) \pi,(2 n-1) \pi}, n=1,2, \ldots$, and has exactly two roots in the strips $\Gamma_{(2 n-1) \pi, 2 n \pi}, n=1,2, \ldots$.

Proof. The lemma follows from calculating the change in argument of $g(Z) \pm C$ on the contours $\Gamma_{n \pi}=\{Z=n \pi+i Y:-\infty<Y<+\infty\}$. Let

$$
g_{n}(Y)=g(n \pi+i Y)=(-1)^{n} \frac{(Y+n \pi i) \sinh (\pi Y)}{n^{2} \pi^{2}+Y^{2}} \text {. }
$$


The change in argument of $g_{0}(Y) \pm C$ is 0 ; the change in argument of $g_{2 k-1}(Y)-$ $C$ is 0 and the change in argument of $g_{2 k}(Y)-C$ is $-2 \pi$; in contrast, the change in argument of $g_{2 k}(Y)+C$ is 0 and the change in argument of $g_{2 k-1}(Y)+C$ has change in argument $-2 \pi$. Taking into account the change in argument of $g(X \pm i \infty) \pm C$, the Argument Principle gives the lemma.

We denote by $\gamma_{\text {crit }}$ the point where the minimum value of $g(t)$ on $[0,2 \pi]$ occurs; $\tan \gamma_{\text {crit }}=\gamma_{\text {crit }} ; \gamma_{\text {crit }} \approx 257^{\circ} 27^{\prime}$.

Lemma 6.2. Consider the equation

$$
g_{\alpha, \gamma}(z)=\frac{\sin \gamma z}{\gamma z}-\alpha \frac{\sin \gamma}{\gamma}=0, \quad z \in \Gamma_{0,1} .
$$

(1) Let $\alpha=1$. For $0<\gamma \leq \gamma_{\text {crit }}$, the equation (6-4) has no roots in $\Gamma_{0,1}$; for $\gamma_{\text {crit }}<\gamma<2 \pi$ there is a single root $z_{0}(1, \gamma) \in \Gamma_{0,1}$ which decreases monotonically from 1 to $\frac{1}{2}$ as $\gamma$ increases from $\gamma_{\text {crit }}$ to $2 \pi$.

(2) Let $-1 \leq \alpha<1$. For $0<\gamma \leq \pi$, the equation (6-4) has no roots in $\Gamma_{0,1}$, for $\pi<\gamma<2 \pi$ there is a single root $z_{0}(\alpha, \gamma) \in \Gamma_{0,1}$ which, for fixed $\alpha$, decreases monotonically from 1 to $\frac{1}{2}$ as $\gamma$ increases from $\pi$ to $2 \pi$.

Proof. The stated roots are understood easily by sketching the graph of $g$ on $[0,2 \pi]$. That there are no complex roots follows from Lemma 5.1.

We are now ready to announce the zeroes of $\operatorname{det}\left(\operatorname{Smbl}^{\frac{1}{p}}\left(\mathbf{K}_{\mathrm{T}}^{+}\right)\right)$. First observe that if $b=0$, we have that $g_{\mathrm{T}}^{+-}$and $g_{\mathrm{T}}^{-+}$are identically 0 ; in particular $\operatorname{Smbl}^{\frac{1}{p}}\left(\mathbf{K}_{\mathbf{T}}^{+}\right)\left(\frac{1}{p} \pm i \infty\right)$ has rank 2 ; this shows that the boundary operator $\mathbf{T}(\mathbf{u}) \vec{\nu}$ does not cover $L$. The following theorem summarizes the roots of $\operatorname{det}\left(\operatorname{Smbl}^{\frac{1}{p}}\left(\mathbf{K}_{\mathbf{T}}^{+}\right)\right)=0$ in $\Gamma_{0,1}$.

Theorem 8. (1) For $t=0$ :

$$
\operatorname{det}\left(\operatorname{Smbl}^{\frac{1}{p}} \mathbf{K}_{\{\cdot\}}^{+}\right)=\frac{1}{\sin ^{4} \pi z} g_{\{\cdot\}}^{++}(z) g_{\{\cdot\}}^{+-}(z) g_{\{\cdot\}}^{-+}(z) g_{\{\cdot\}}^{--}(z) .
$$

(2) The equations $g_{\mathbf{T}}^{++}=0$ and $g_{\mathbf{N}}^{--}=0$ have roots where

$$
\frac{\sin (2 \pi-\theta) z}{2 \pi-\theta}=\frac{b}{2-b} \frac{\sin (2 \pi-\theta)}{2 \pi-\theta} \text {; }
$$

Equation (6-6) has a root $z_{0}$ in $\Gamma_{0,1}$ for $0<\theta<\pi \quad(0 \leq b<1)$, or for only $0<\theta<2 \pi-\gamma_{\text {crit }}(b=1)$.

(3) The equations $g_{\mathbf{T}}^{--}=0$ and $g_{\mathbf{N}}^{++}=0$ have roots where

$$
\frac{\sin (2 \pi-\theta) z}{(2 \pi-\theta) z}=-\frac{b}{2-b} \frac{\sin (2 \pi-\theta)}{2 \pi-\theta} \text {. }
$$

Equation (6-7) has a root $z_{0}$ in $\Gamma_{0,1}$ for $0<\theta<\pi \quad(0 \leq b \leq 1)$. 
(4) The equation $g_{\mathrm{T}}^{+-}=0$ has a root where

$$
\frac{\sin \theta z}{\theta z}=-\frac{\sin \theta}{\theta} \text {. }
$$

Equation (6-8) has a root $z_{0}$ in $\Gamma_{0,1}$ iff $\pi<\theta<2 \pi$.

(5) The equation $g_{\mathrm{T}}^{-+}=0$ has a root where

$$
\frac{\sin \theta z}{\theta z}=\frac{\sin \theta}{\theta} \text {. }
$$

Equation (6-9) has a root $z_{0}$ in $\Gamma_{0,1}$ iff $2 \pi-\gamma_{\text {crit }}<\theta<2 \pi$.

(6) The equation $g_{\mathbf{N}}^{+-}=0$ has a root where

$$
\frac{\sin \theta z}{\theta z}=-\frac{b}{2+b} \frac{\sin \theta}{\theta} \text {. }
$$

Equation (6-10) has a root $z_{0}$ in $\Gamma_{0,1}$ iff $\pi<\theta<2 \pi$.

(7) The equation $g_{\mathbf{N}}^{-+}=0$ has a root where

$$
\frac{\sin \theta z}{\theta z}=\frac{b}{2+b} \frac{\sin \theta}{\theta} \text {. }
$$

Equation (6-11) has a root $z_{0}$ in $\Gamma_{0,1}$ iff $\pi<\theta<2 \pi$.

(8) If $0<b \leq 1$, for $0<\frac{1}{p} \leq \frac{1}{2}$ the change in argument of $\operatorname{det}\left(\mathrm{Smbl}^{\frac{1}{p}} \mathbf{K}_{\{\cdot\}}^{+}\right)$ on the contour $\Gamma_{\frac{1}{p}}$ is 0 .

(9) If $0<b \leq 1$, when $\theta=\pi$, for $0<\frac{1}{p}<1$ the change in argument of $\operatorname{det}\left(\mathrm{Smbl}^{\frac{1}{p}} \mathbf{K}_{\{\cdot\}}^{+}\right)$on the contour $\Gamma_{\frac{1}{p}}$ is 0 .

Proof. Statement (1) is Theorem 6; statements (2)-(7) follow from Lemma 5.2. Statements (8) and (9) are proved by calculating the change in argument near $\frac{1}{p}=0$ and the Argument Principle.

Remark. At the zeroes of $\operatorname{det}\left(\operatorname{Smbl}^{\frac{1}{p}} \mathbf{K}_{\{\cdot\}}^{+}\right)$the eigenvectors of the the $2 \times 2$ matrices $A^{ \pm}$are easily computed; in turn the eigenvectors of $\hat{U} \tilde{\mathbf{K}}_{\{\cdot\}}^{+} \hat{U}$ and $\tilde{\mathbf{K}}_{\{\cdot\}}^{+}$are calculated.

Definition 6.1. With $\mathbf{K}_{\{\cdot\}}^{ \pm}$as in equation (4-28), for $\frac{1}{p}$ not a zero of $\operatorname{det}\left(\sin \pi z \operatorname{Smbl}^{\frac{1}{p}}\left(\mathbf{K}_{\{\cdot\}}^{ \pm}\right)\right)$, define

$$
I_{\{\cdot\}}^{ \pm}\left(\frac{1}{p}, b, \theta\right)=\left[\text { number of zeroes of } \operatorname{det}\left(\sin \pi z \operatorname{Smbl}^{\frac{1}{p}}\left(\mathbf{K}_{\{\cdot\}}^{ \pm}\right)\right) \text {in }\left(0, \frac{1}{p}\right)\right] \text {. }
$$

We note the following facts about $I_{\{\cdot\}}^{ \pm}\left(\frac{1}{p}, b, \theta\right)$.

(1) $I_{\{\cdot\}}^{ \pm}\left(\frac{1}{p}, b, \theta\right)=\frac{1}{2 \pi}$ (change in arg of $\operatorname{det} \tilde{\mathbf{K}}_{\{\cdot\}}^{ \pm}$on $\Gamma_{\frac{1}{p}}$ ).

(2) $I_{\{\cdot\}}^{+}\left(\frac{1}{p}, b, \theta\right)=I_{\{\cdot\}}^{-}\left(\frac{1}{p}, b, 2 \pi-\theta\right)$.

(3) For $0<\theta<\pi, I_{\mathrm{T}}^{+}\left(\frac{1}{p}, b, \theta\right)=I_{\mathbf{N}}^{+}\left(\frac{1}{p}, b, \theta\right)$.

(4) For $\pi<\theta<2 \pi, I_{\mathrm{T}}^{+}\left(\frac{1}{p}, b, \theta\right)=I_{\mathrm{T}}^{-}\left(\frac{1}{p}, b, 2 \pi-\theta\right)$ is independent of $b$ for $0<b \leq 1$. 
Let us now return to the problem on the domain $\Omega^{+}$as described in $\S 4$. For $\mathbf{f} \in L^{p}\left(\partial \Omega^{+}\right)$, let

$$
\begin{aligned}
& \mathbf{K}_{\mathbf{T}}^{ \pm} \mathbf{f}(P)= \pm \mathbf{I f}(P)+\text { p.v. } \int_{\partial \Omega^{+}} \mathbf{T}_{\vec{\nu}(Q)}(\boldsymbol{\Gamma}(X-Q)) \mathbf{f}(Q) d \sigma_{Q}, \\
& \mathbf{K}_{\mathbf{T}}^{ \pm} \mathbf{f}(P)= \pm \mathbf{I f}(P)+\text { p.v. } \int_{\partial \Omega^{+}} \mathbf{N}_{\vec{\nu}(Q)}(\boldsymbol{\Gamma}(X-Q)) \mathbf{f}(Q) d \sigma_{Q} .
\end{aligned}
$$

When (6-13) or (6-14) is written as a big $4 N \times 4 N$ system of Mellin operators as in (3-1) ff., the operators $K^{(2 i)}$ of (3-3) correspond to the operator $\mathbf{K}_{\{\cdot\}}^{ \pm}$of (4-28) with $\theta=\theta_{2 i}$; the operators $K^{(2 i-1)}$ of (3-3) correspond to the operator $\mathbf{K}_{\{\cdot\}}^{ \pm}$of (4-28) with $\theta=\pi$. Using Theorem 2, Theorem 7, and Theorem 8, we obtain

Theorem 9. Let $\mathbf{K}_{\{\cdot\}}^{ \pm}$denote one of the operators (6-13) or (6-14). Then

(1) For $1<p<\infty, \mathbf{K}_{\{\cdot\}}^{ \pm}$is a Fredholm operator on $L^{p}\left(\partial \Omega^{+}\right)$iff for all $j, j=1, \ldots, N$, the operators (4-28), with $\theta=\theta_{2 j}$, is a Fredholm operator on $\left[L^{p}\left(\mathbf{R}^{+}\right)\right]^{4}$.

(2) If $b=0, \mathbf{K}_{\mathbf{T}}^{ \pm}$is not a Fredholm operator on $L^{p}\left(\partial \Omega^{+}\right)$for any $p$, $1<p<\infty$.

(3) If $b=0, \mathbf{K}_{\mathbf{N}}^{ \pm}$is not a Fredholm operator on $L^{p}\left(\partial \Omega^{+}\right)$iff for some $j$, $j=1, \ldots, N, \sin \left(\theta_{2 j} \frac{1}{p}\right)=0$ or $\sin \left(\left(2 \pi-\theta_{2 j}\right) \frac{1}{p}\right)=0$.

(4) If $0<b \leq 1, \mathbf{K}_{\{\cdot\}}^{ \pm}$is a Fredholm operator on $L^{p}\left(\partial \Omega^{+}\right)$for all $p$, $2 \leq p<\infty$.

(5) If $0<b \leq 1$, the "bad values" of $p$ in $(1,2)$, for which the operators $\mathbf{K}_{\{\cdot\}}^{ \pm}$are not Fredholm on $L^{p}\left(\partial \Omega^{+}\right)$form a discrete set of cardinality at most $2 N$.

(6) If $p$ is a "good value" for which $\mathbf{K}_{\{\cdot\}}^{ \pm}$is a Fredholm operator on $L^{p}\left(\partial \Omega^{+}\right)$, the index of $\mathbf{K}_{\{\cdot\}}^{ \pm}$on $L^{p}\left(\partial \Omega^{+}\right)$is given by

$$
\operatorname{ind}_{p}\left(\mathbf{K}_{\{\cdot\}}^{ \pm}\right)=\sum_{j=1}^{N} I_{\{\cdot\}}^{ \pm}\left(\frac{1}{p}, b, \theta_{2 j}\right) .
$$

Proof. The determinant of the symbols of (6-13) and (6-14) are calculated using Theorem 2. Statements (1), (2), and (3) follow from the formulas (5-13) and (5-14). Statements (4) and (5) follow from Theorem 2, statements (8) and (9), applied to the operators (4-28). Statement (6) is the Index Theorem, Theorem 1.

Remarks. When uniqueness is shown for a double layer potential on $L^{2}\left(\partial \Omega^{+}\right)$, for the "good values" of $p$ the index on $L^{p}\left(\partial \Omega^{+}\right)$is the dimension of the kernel since uniqueness for the adjoint holds in $L^{q}\left(\partial \Omega^{+}\right), 2 \leq q<\infty$. 
In contrast to the case of a finite interval, for the "good values" of $p$, the operators (4-28) have index $=0$ on $\left[L^{p}\left(\mathbf{R}^{+}\right)\right]^{4}$. Cf. [E] or [LP, Definition 3.2] for the correct notion of principal symbol in this case; the change in argument of $\operatorname{det}\left(\operatorname{Smbl}^{\frac{1}{p}} \mathbf{K}_{\{\cdot\}}^{ \pm}\right)$at $t=0$ is killed by the change in argument at $t=\infty$.

\section{REFERENCES}

[C] M. Costabel, Singular integral operators on curves with corners, Integral Equations and Operator Theory 3 (1980), 323-349.

[DKV] B. E. J. Dahlberg, C. E. Kenig, and G. C. Verchota, Boundary value problems for the systems of elastostatics in Lipschitz domains, Duke Math. J. 57 (1988), 795-818.

[E] J. Elschner, Asymptotics of solutions of pseudodifferential equations of Mellin type, Math. Nachr. 130 (1987), 267-305.

[FKV] E. B. Faves, C. E. Kenig, and G. C. Verchota, The Dirichlet problem for the Stokes system on Lipschitz domain, Duke Math. J. 57 (1988), 769-795.

[La] O. A. Ladyzhenskaya, The mathematical theory of viscous incompressible flow, Gordon and Breach, 1963.

[LP] J. E. Lewis and C. Parenti, Pseudodifferential operators of Mellin type, Comm. Partial Differential Equations 8 (1983), 477-544.

[F] C. J. Karal and S. N. Karp, The elastic-field behavior in the neighborhood of a crack of arbitrary angle, Comm. Pure Appl. Math. 15 (1962), 413-421.

$[\mathrm{Ku}] \quad$ V. D. Kupradze, Potential methods in the theory of elasticity, Israel Program for Scientific Translations, Jerusalem, 1965.

[St] E. M. Stein, Singular integrals and differentiability properties of functions, Princeton Univ. Press, 1966.

[V] D. Vasilopoulos, On the determination of higher order terms of singular elastic stress fluids near corners, Numer. Math. 53 (1988), 51-96.

Department of Mathematics, University of Illinois at Chicago, P.O. Box 4348, CHICAGO, ILLINOIS 60680-4348 (U12585@UICVM.BITNET) 